\title{
Stroke awareness decreases prehospital delay after acute ischemic stroke in korea
}

\author{
Young Seo Kim², Sang-Soon Park², Hee-Joon Bae ${ }^{3}$, A-Hyun Cho ${ }^{4}$, Yong-Jin Cho ${ }^{5}$, Moon-Ku Han ${ }^{3}$, Ji Hoe Heo ${ }^{6}$, \\ Kyusik Kang ${ }^{7}$, Dong-Eog Kim ${ }^{8}$, Hahn Young Kim ${ }^{9}$, Gyeong-Moon Kim ${ }^{10}$, Sun Uk Kwon ${ }^{11}$, Hyung-Min Kwon ${ }^{12}$, \\ Byung-Chul Lee ${ }^{13}$, Kyung Bok Lee ${ }^{14}$, Seung-Hoon Lee ${ }^{2}$, Su-Ho Lee ${ }^{15}$, Yong-Seok Lee ${ }^{12}$, Hyo Suk Nam6, \\ Mi-Sun Oh ${ }^{13}$, Jong-Moo Park, Joung-Ho Rha ${ }^{16}$, Kyung-Ho Yu ${ }^{13}$, Byung-Woo Yoon ${ }^{2 *}$
}

\begin{abstract}
Background: Delayed arrival at hospital is one of the major obstacles in enhancing the rate of thrombolysis therapy in patients with acute ischemic stroke. Our study aimed to investigate factors associated with prehospital delay after acute ischemic stroke in Korea.

Methods: A prospective, multicenter study was conducted at 14 tertiary hospitals in Korea from March 2009 to July 2009. We interviewed 500 consecutive patients with acute ischemic stroke who arrived within 48 hours. Univariate and multivariate analyses were performed to evaluate factors influencing prehospital delay.

Results: Among the 500 patients (median 67 years, 62\% men), the median time interval from symptom onset to arrival was 474 minutes (interquartile range, 170-1313). Early arrival within 3 hours of symptom onset was significantly associated with the following factors: high National Institutes of Health Stroke Scale (NIHSS) score, previous stroke, atrial fibrillation, use of ambulance, knowledge about thrombolysis and awareness of the patient/ bystander that the initial symptom was a stroke. Multivariable logistic regression analysis indicated that awareness of the patient/bystander that the initial symptom was a stroke (OR 4.438,95\% Cl 2.669-7.381), knowledge about thrombolysis (OR 2.002, 95\% Cl 1.104-3.633) and use of ambulance (OR 1.961, 95\% Cl 1.176-3.270) were significantly associated with early arrival.

Conclusions: In Korea, stroke awareness not only on the part of patients, but also of bystanders, had a great impact on early arrival at hospital. To increase the rate of thrombolysis therapy and the incidence of favorable outcomes, extensive general public education including how to recognize stroke symptoms would be important.
\end{abstract}

\section{Background}

Despite current evidences supporting the time extension of intravenous recombinant tissue plasminogen activator (rt-PA) therapy in acute ischemic stroke patients, it is well known that early administration of thrombolytics is beneficial for patient outcome [1]. Since intravenous rt-PA is the only approved treatment for acute ischemic stroke, shortening the time between symptom onset and hospital arrival is important. Although, many interventions to reduce prehospital delay have been conducted,

\footnotetext{
* Correspondence: bwyoon@snu.ac.kr

${ }^{2}$ Department of Neurology, Seoul National University Hospital, Seoul, Republic of Korea

Full list of author information is available at the end of the article
}

$3-8.5 \%$ of all stroke patients receive thrombolytic therapy in the US [2] and only $2.1 \%$ in Korea [3].

There have been numerous studies of the factors associated with prehospital delay and some factors such as contacting the primary physician, or not using Emergency Medical Services (EMS), were in almost all cases found to be associated with delayed arrival time $[4,5]$. However, findings concerning the impact of demographics and clinical factors, as well as of knowledge about stroke, were somewhat inconsistent, perhaps due to differences in location, time of investigation and medical environment. In one study, perceptual, social and behavioral factors, rather than knowledge, were suggested to be important for decreasing arrival delay [6], and the role of the bystander in delivery of acute
C Biomed Central

(c) 2011 Kim et al; licensee BioMed Central Ltd. This is an Open Access article distributed under the terms of the Creative Commons Attribution License (http://creativecommons.org/licenses/by/2.0), which permits unrestricted use, distribution, and reproduction in any medium, provided the original work is properly cited. 
stroke was highlighted. South Korea has a unique culture in which family relationships tend to be close and are held in high regard, and where relatives often live together. In addition, knowledge about acute stroke treatment is not widespread, especially among the elderly and people with little education [7]. Therefore, the results of large studies in other countries may not be applicable in Korea, and it is likely that rapid reactions on the part of bystander after the onset of stroke symptoms may be extremely important.

The aims of this study were to identify factors that influence hospital arrival time after an acute ischemic stroke, and to investigate whether awareness of stroke symptoms not only on the part of patient but also of the bystander, has a significant impact on reducing prehospital delay.

\section{Methods}

This study was designed as a prospective, multicenter, consecutive characterization, which was conducted at 14 tertiary hospitals in Seoul, Korea, and the surrounding metropolitan area. This area has a population of approximately 24 million, and study hospitals were selected to represent different geographic locations that were primarily responsible for all stroke patients in these locations. The inclusion criteria were patients with neurologic symptoms who were hospitalized at the study hospitals and diagnosed with non-traumatic ischemic stroke by diffusion magnetic resonance imaging (MRI). Patients who visited the hospital within 48 hours of symptom onset, and were above 19 were recruited. Exclusion criteria were diagnoses of intracerebral hemorrhage, subarachnoid hemorrhage, in-hospital stroke, or lesion-negative transient ischemic attack (TIA) and patients who had been treated by thrombolysis before visiting the study hospitals. There were no public campaigns or educational efforts before or during the study. The study was approved by the institutional review board (IRB) of each hospital.

The study was performed from March to July 2009, and we decided to stop recruiting when the total number of patients reached 500. At each hospital, all of the patients who arrived at the Emergency Department (ED) and were admitted to the stroke unit were reviewed to confirm eligibility. When patients were deemed eligible, research nurses who had been formally trained in standardized definitions and data collection techniques asked them to participate in the study. The patients were interviewed within 48 hours of admission after providing informed consent. Patients who did not consent to an interview were excluded. If a patient was not able to communicate, a bystander who had witnessed the patient's symptom onset and could describe the exact arrival process was interviewed. However, patients who died soon after admission or were hospitalized in the intensive care unit were excluded because they were not available for interview. Demographic characteristics and circumstances from the onset of symptoms to arrival at the study hospital were recorded, and clinical information about the patients, such as the National Institute of Health Stroke Scale (NIHSS), was obtained from medical records. Patients were registered consecutively using the web-based database electronic Case Reporting Form (e-CRF), which was available at all the study hospitals.

Prehospital delay was defined as the time from symptom onset to arrival at the ED of the study hospital. If the symptoms occurred during sleep, the time of awakening was recorded as the time of onset because it represented the time when medical help could be sought. For patients who were referred by other hospitals or primary care physicians, arrival times and transportation methods to the referral hospital were also recorded.

Patient baseline characteristics were represented by median (interquartile range), number and proportion values. Because the distribution of the prehospital delay times was positively skewed, time differences according to explanatory variables were represented by median and $25^{\text {th }}$ and $75^{\text {th }}$ percentile values, and the MannWhitney $U$ test was used for univariate analysis. Patients were then divided into an early arrival group $(\leq 3$ hours) and a late arrival group ( $>3$ hours), and the explanatory variables were compared by Pearson's $\chi^{2}$ test and the Mann-Whitney $U$ test. Because current evidence supports a change in the thrombolysis indication time from 3 to 4 hours 30 minutes, we thought it reasonable to dichotomize by 3 -hour periods. Finally, multivariable logistic regression analysis was used to analyze the factors independently associated with prehospital delay. Explanatory variables, which were identified by univariate analysis at $\mathrm{P}<0.2$, were selected and entered into the models. All significance tests were 2-tailed, and differences were considered to be statistically significant at $\mathrm{P}<0.05$. Data were analyzed with SPSS version 12.0 for Windows (SPSS Inc).

\section{Results}

Of the 500 patients who were registered in the study, $62 \%$ were male, and the median age was 67 . The median prehospital delay time was 474 minutes (interquartile range, 170 to 1313 minutes). One hundred and two patients (21\%) arrived within 2 hours, 131 (26\%) within 3 hours and 215 (43\%) within 6 hours. Thirty patients (6\%) underwent thrombolysis. Table 1 shows the baseline characteristics of the study population and the frequencies of the factors considered as explanatory variables for delayed arrival. One hundred and sixty-nine 
Table 1 Baseline characteristics of patients

\begin{tabular}{|c|c|}
\hline Characteristics & \\
\hline Age, years & $67(57-73)^{*}$ \\
\hline Gender, male (\%) & $308(62)$ \\
\hline Time to hospital arrival, minutes & $474(170-1313)^{*}$ \\
\hline NIHSS, score & $3(2-6)^{*}$ \\
\hline \multicolumn{2}{|l|}{ Risk factors } \\
\hline Previous stroke (\%) & $116(23)$ \\
\hline Hypertension (\%) & $316(63)$ \\
\hline Diabetes (\%) & $134(27)$ \\
\hline Hyperlipidemia (\%) & $72(14)$ \\
\hline Current smoker (\%) & $150(30)$ \\
\hline Atrial fibrillation (\%) & $44(9)$ \\
\hline Coronary heart disease (\%) & $38(8)$ \\
\hline Family history of stroke (\%) & $147(29)$ \\
\hline Study hospital located in Seoul (\%) & $324(65)$ \\
\hline \multicolumn{2}{|l|}{ Education } \\
\hline Low (0-6 years) (\%) & $169(34)$ \\
\hline Medium (6-12 years) (\%) & $212(42)$ \\
\hline High ( $\geq 12$ years) (\%) & $116(23)$ \\
\hline Living alone (\%) & $66(13)$ \\
\hline Visit hospital regularly (\%) & $338(68)$ \\
\hline Presence of bystander at time of symptom onset (\%) & $355(71)$ \\
\hline Arrival through referral (\%) & $169(34)$ \\
\hline \multicolumn{2}{|l|}{ Mode of transport } \\
\hline Ambulance $(\%)$ & $182(36)$ \\
\hline Personal vehicle (\%) & $200(40)$ \\
\hline Public transportation (\%) & $113(23)$ \\
\hline Knowledge by patient of thrombolysis (\%) & $92(18)$ \\
\hline Awareness of the patient/bystander that the initial symptom was stroke related (\%) & $179(36)$ \\
\hline
\end{tabular}

$\mathrm{N}=500$.

*Median $\left(25^{\text {th }}-75^{\text {th }}\right.$ percentile).

patients (34\%) were referred from other hospitals and 182 (36\%) used ambulances. The majority of cases occurred at home (71\%), and 145 (29\%) patients were alone at the time of symptom onset. Only $92(18 \%)$ patients had knowledge of thrombolysis. The media and family/neighbors made up the largest sources of information (Figure 1). Of the 92 patients who knew about thrombolysis, 80 (87\%) replied that it should be performed as soon as possible or within 3 hours. Stroke awareness on the part of patient or bystander was present in only 179 cases (36\%), and $140(28 \%)$ patients responded that they had never heard of strokes. When educational level was analyzed according to knowledge of thrombolysis and stroke awareness, highly educated patients ( $\geq 12$ years) had more and correct knowledge than patients with low to medium education $(31.9 \%$ versus $13.9 \%, \mathrm{P}<0.001)$. However, stroke awareness was not different by educational status (33.6\% versus $36.2 \%, P=0.609$ ).

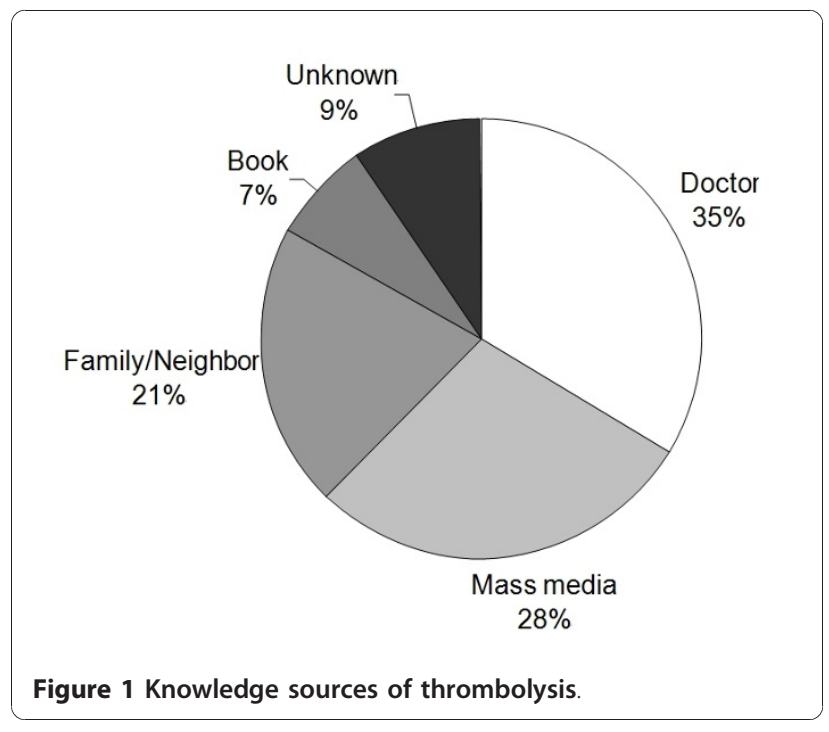


Table 2 presents prehospital delay time by explanatory variables such as NIHSS score, risk factors and general patient characteristics. Shorter times were significantly associated with high NIHSS scores, atrial fibrillation, use of an ambulance, and awareness by patient/bystander that the initial symptom was stroke-related. Diabetes, hyperlipidemia and referral from other hospitals were associated with longer times.

Analysis of variables according to arrival time (Table 3) showed that previous stroke history and knowledge about thrombolysis were also related to early arrival. However, diabetes and hyperlipidemia were no longer significantly associated with late arrival.

Multivariable logistic regression analysis (Table 4) identified the variables that were independently associated with early arrival. Among them, awareness by the patient/bystander that the initial symptom was strokerelated was highly associated with early arrival (OR, 4.438; 95\% CI, 2.669-7.381). Knowledge about thrombolysis (OR, 2.002; 95\% CI, 1.104-3.633), use of ambulance (OR, 1.961; 95\% CI, 1.176-3.270) and high NIHSS score (OR, 1.101; 95\% CI, 1.038-1.167) were also independently associated with early arrival, and referral from another hospital (OR, 0.116; CI, 0.059-0.228) was independently associated with late arrival.

\section{Discussion}

Despite the results of previous studies that showed that early arrival is not associated with patients' knowledge about stroke [8-10], it is still believed that awareness of stroke as a severe symptom may lead to shorter delay times and increased thrombolysis rates [11,12]. In our study, stroke awareness and knowledge about thrombolysis were independently associated with lower prehospital delay. Interestingly, awareness of the patient/ bystander that the initial symptom was stroke-related was the factor most strongly associated with early arrival. This result may suggest that an immediate response not only by the patient, but also by the bystander, is important for early arrival. However, only 178 patients (36\%), even including bystanders' knowledge, knew that the patient had had a stroke, and only 92 patients (18\%) had knowledge of thrombolysis. Even though these results may appear disappointing, the opportunity exists to increase public knowledge and thrombolysis rates. Furthermore, the finding that only one-third of patients had heard of thrombolysis from their doctors was surprising, and doctors in Korea should make greater efforts to educate patients.

Our study confirmed results from previous reports that educational level was not associated with early arrival $[13,14]$. However, we could speculate that the bystander's educational level may potentially affect early arrival. In our data, highly educated patients were more informed about thrombolysis, and patients who had knowledge about thrombolysis presented to the ED earlier. Nevertheless, patients' educational level was not associated with early arrival. It may be that statistical significance was lost because the patients who were accompanied by highly educated bystanders arrived faster because of the bystanders' knowledge of stroke symptoms. Therefore, we suggest that the bystander's knowledge and behavior after stroke onset may greatly impact early arrival. Because there are many elderly people in Korea with little education who live together with their descendants, the knowledge and behavior of bystanders may be more important than those of patients.

Similar to many other studies $[8,9,15-18]$, we found that severe stroke and use of ambulance were significantly associated with early arrival, and referral from other hospitals was negatively associated with it. We also found that patients who were referred from other hospitals never used EMS when they visited primary care centers. Interestingly, 37 patients who were referred by primary Oriental physicians had longer prehospital delay times (median, 1214 minutes) than patients who came from Western physicians (median, 730 minutes). Considering that patients who arrived after 48 hours were not included in this study, the delay time of patients who were referred by primary Oriental physicians may actually be greater. In Korea, traditional Oriental medicine is very familiar to the general public, especially to the elderly and subjects with little education [7]. In order to increase the thrombolysis rate and better stroke outcome, publicity focusing on these subjects is needed.

Demographic and clinical factors such as age, sex, premorbid disability, risk factors, living alone, visiting the hospital regularly, and presence of a bystander, were not associated with early arrival in multivariate analyses, consistent with other reports [19-21]. In one study, recognition of symptoms by a witness was related to early arrival [21], but in our data, presence of a bystander on its own did not have any significance. These findings may indicate that the presence of witnesses as well as their knowledge and behavior are important. Care sought after stroke symptom onset was associated with the previous stroke history of patients in one study [22], but it did not remain significant after multivariate analysis, suggesting that more education of patients during hospitalization is required.

Although this study was conducted prospectively in 14 tertiary hospitals, it has a few limitations. First, although Seoul and its metropolitan area include almost half the population of Korea, it includes few rural regions. In fact, the metropolitan area in this study consists of more than 1 million people, and their socio-economic 
Table 2 Factors associated with increased time from symptom onset to hospital arrival: univariate analysis

\begin{tabular}{|c|c|c|c|}
\hline Variables & $\mathrm{N}(\%)$ & Prehospital delay & $\mathbf{P}$ \\
\hline \multicolumn{4}{|l|}{ Clinical } \\
\hline NIHSS, score & & & $<0.001$ \\
\hline $0-3$ & $261(52)$ & $595(242-1452)$ & \\
\hline $4-6$ & $136(27)$ & $573(244-1357)$ & \\
\hline$\geq 7$ & $103(21)$ & $170(55-508)$ & \\
\hline Pre-morbid disability $(\mathrm{mRS}>2)$ & & & 0.798 \\
\hline Yes & $18(4)$ & $463(126-1196)$ & \\
\hline No & $482(96)$ & $471(170-1308)$ & \\
\hline \multicolumn{4}{|l|}{ Risk factors } \\
\hline Previous stroke & & & 0.088 \\
\hline Yes & $116(23)$ & $316(110-1241)$ & \\
\hline No & $384(77)$ & $502(210-1338)$ & \\
\hline Hypertension & & & 0.632 \\
\hline Yes & $316(63)$ & $540(165-1305)$ & \\
\hline No & $184(37)$ & $405(170-1317)$ & \\
\hline Diabetes & & & 0.049 \\
\hline Yes & $134(27)$ & $568(234-1460)$ & \\
\hline No & $366(73)$ & $449(148-1253)$ & \\
\hline Hyperlipidemia & & & 0.039 \\
\hline Yes & $72(14)$ & $753(236-1391)$ & \\
\hline No & $428(76)$ & $432(152-1297)$ & \\
\hline Current smoker & & & 0.637 \\
\hline Yes & $150(30)$ & $466(196-1227)$ & \\
\hline No & $350(70)$ & $504(150-1344)$ & \\
\hline Atrial fibrillation & & & 0.002 \\
\hline Yes & $44(9)$ & 215 (59-948) & \\
\hline No & $456(91)$ & 521 (194-1328) & \\
\hline Coronary heart disease & & & 0.067 \\
\hline Yes & $38(8)$ & $277(69-1012)$ & \\
\hline No & $462(92)$ & $504(180-1314)$ & \\
\hline Family history of stroke & & & 0.171 \\
\hline Yes & $147(29)$ & 762 (327-1409) & \\
\hline No & $353(71)$ & $682(230-1353)$ & \\
\hline \multicolumn{4}{|l|}{ General } \\
\hline Location of study hospital & & & 0.096 \\
\hline Seoul & $324(65)$ & $558(165-1365)$ & \\
\hline Metropolitan area & $176(35)$ & $397(170-1126)$ & \\
\hline Education & & & 0.572 \\
\hline Low (0-6 years) & $169(34)$ & $444(170-1294)$ & \\
\hline Medium (6-12 years) & $215(43)$ & $551(210-1308)$ & \\
\hline High ( $\geq 12$ years) & $116(23)$ & $379(122-1307)$ & \\
\hline Living alone & & & 0.322 \\
\hline Yes & $435(87)$ & $506(283-1348)$ & \\
\hline No & $65(13)$ & $462(150-1309)$ & \\
\hline Visit hospital regularly & & & 0.503 \\
\hline Yes & $338(68)$ & $504(152-1248)$ & \\
\hline No & $162(32)$ & $466(183-1350)$ & \\
\hline Presence of bystander at time of symptom onset & & & 0.742 \\
\hline Yes & $145(29)$ & $502(170-1358)$ & \\
\hline No & $355(71)$ & $470(170-1252)$ & \\
\hline Arrival through referral & & & $<0.001$ \\
\hline
\end{tabular}


Table 2 Factors associated with increased time from symptom onset to hospital arrival: univariate analysis (Continued)

\begin{tabular}{|c|c|c|c|}
\hline Yes & $169(34)$ & $847(355-1575)$ & \\
\hline No & $331(66)$ & $347(106-1015)$ & \\
\hline Mode of transport & & & $<0.001$ \\
\hline Ambulance & $182(36)$ & $301(79-758)$ & \\
\hline Personal vehicle & $205(41)$ & $608(242-1373)$ & \\
\hline Public transportation & $113(23)$ & $858(290-1620)$ & \\
\hline Knowledge by patient of thrombolysis & & & 0.132 \\
\hline Yes & $92(18)$ & $349(113-1121)$ & \\
\hline No & $408(82)$ & $502(208-1328)$ & \\
\hline Awareness of the patient/bystander that the initial symptom was stroke related & & & $<0.001$ \\
\hline Yes & $179(36)$ & 260 (100-708) & \\
\hline No & $321(64)$ & 650 (290-1458) & \\
\hline
\end{tabular}

Values are median $\left(25^{\text {th }}-75^{\text {th }}\right.$ percentile) and are given in minutes.

*Mann-Whitney $\mathrm{U}$ test or Kruskal-Wallis test was used.

status is not very different from that of the patients in Seoul. Therefore, there might be some economic, educational and socio-psychological factor biases. Second, we excluded patients who could not be interviewed (severe symptoms, death), and those who received thrombolysis therapy outside the hospital. These exclusion criteria may lead to slightly increased delay times and decreased median NIHSS scores, because patients with severe symptoms tend to arrive earlier. In addition, we only included patients who arrived within 48 hours from symptom onset, and this may also affect the overall delay time. However, we only investigated patients who arrived within 48 hours because they are more likely to be candidates for thrombolysis.

\section{Conclusion}

This is the first multicenter study to investigate factors associated with prehospital delay in Korea and to find

Table 3 Distribution of clinical characteristics according to arrival time

\begin{tabular}{|c|c|c|c|}
\hline Characteristics & $\begin{array}{l}\text { Early arrival ( } \leq 3 \text { hours) } \\
(\mathrm{n}=132)\end{array}$ & $\begin{array}{l}\text { Late arrival (>3 hours) } \\
\qquad(\mathrm{n}=368)\end{array}$ & $P$ \\
\hline Age, years & $68(57-73)^{+}$ & $66(57-74)^{\dagger}$ & $1.000^{*}$ \\
\hline Gender, male (\%) & $80(61)$ & $228(62)$ & 0.784 \\
\hline NIHSS, score & $5(2-10)^{\dagger}$ & $3(1-5)^{\dagger}$ & $<0.001^{*}$ \\
\hline Pre-morbid disability $(m R S>2)(\%)$ & $7(5)$ & $11(3)$ & 0.232 \\
\hline \multicolumn{4}{|l|}{ Risk factors } \\
\hline Previous stroke (\%) & $43(33)$ & $73(20)$ & 0.003 \\
\hline Hypertension (\%) & $85(64)$ & $231(63)$ & 0.740 \\
\hline Diabetes (\%) & $27(21)$ & $107(29)$ & 0.055 \\
\hline Hyperlipidemia (\%) & $13(10)$ & $59(16)$ & 0.083 \\
\hline Current smoker (\%) & $35(27)$ & $115(31)$ & 0.308 \\
\hline Atrial fibrillation (\%) & $21(16)$ & $23(6)$ & 0.001 \\
\hline Coronary heart disease (\%) & $15(11)$ & $23(6)$ & 0.057 \\
\hline Family history of stroke (\%) & $35(27)$ & $112(30)$ & 0.396 \\
\hline Study hospital located in Seoul (\%) & $84(64)$ & $240(65)$ & 0.744 \\
\hline Highly educated ( $\geq 12$ years) $(\%)$ & $36(27)$ & $80(22)$ & 0.196 \\
\hline Living alone (\%) & $11(8)$ & $55(15)$ & 0.054 \\
\hline Visit hospital regularly (\%) & $91(69)$ & $247(67)$ & 0.702 \\
\hline Presence of bystander at time of symptom onset (\%) & $92(70)$ & $263(72)$ & 0.701 \\
\hline Arrival through referral (\%) & $15(11)$ & $154(42)$ & $<0.001$ \\
\hline Arrival by Ambulance (\%) & $71(54)$ & $111(30)$ & $<0.001$ \\
\hline Knowledge by patient of thrombolysis (\%) & $34(26)$ & $58(16)$ & 0.011 \\
\hline Awareness of the patient/bystander that the initial symptom was stroke related (\%) & $75(57)$ & $104(28)$ & $<0.001$ \\
\hline
\end{tabular}

Pearson's $\chi^{2}$ test and the *Mann-Whitney $U$ test were used.

${ }^{+}$Median $\left(25^{\text {th }}-75^{\text {th }}\right.$ percentile). 
Table 4 Multivariable logistic regression analysis: factors independently associated with early arrival

\begin{tabular}{|c|c|c|c|}
\hline & \multicolumn{2}{|c|}{ Arrival time ( $\leq 3$ hours) } & \multirow[b]{2}{*}{$\mathbf{P}^{*}$} \\
\hline & Crude OR $(95 \% \mathrm{Cl})$ & Adjusted OR $(95 \% \mathrm{Cl})$ & \\
\hline NIHSS & $1.152(1.099-1.207)$ & $1.101(1.038-1.167)$ & 0.001 \\
\hline Previous stroke & $1.952(1.251-3.047)$ & $1.185(0.680-2.067)$ & 0.549 \\
\hline Diabetes & $0.627(0.388-1.013)$ & $0.642(0.367-1.122)$ & 0.120 \\
\hline Hyperlipidemia & $0.572(0.303-1.081)$ & $0.485(0.227-1.035)$ & 0.061 \\
\hline Atrial fibrillation & $2.838(1.513-5.323)$ & $2.152(0.975-4.747)$ & 0.058 \\
\hline Coronary heart disease & $1.923(0.971-3.809)$ & $0.882(0.362-2.146)$ & 0.781 \\
\hline Living alone & $1.933(0.979-3.817)$ & $1.710(0.767-3.810)$ & 0.190 \\
\hline Arrival through referral & $0.178(0.100-0.317)$ & $0.116(0.059-0.228)$ & $<0.001$ \\
\hline Arrival by Ambulance & $2.697(1.790-4.063)$ & $1.961(1.176-3.270)$ & 0.010 \\
\hline Knowledge by patient of thrombolysis & $1.854(1.147-2.998)$ & $2.002(1.104-3.633)$ & 0.022 \\
\hline Awareness of the patient/bystander that the initial symptom was stroke related & $3.340(2.211-5.045)$ & $4.438(2.669-7.381)$ & $<0.001$ \\
\hline
\end{tabular}

*P for multivariate model.

Candidate variables were selected from the results of univariate analysis with $P<0.2$.

that awareness of stroke symptoms on the part of patient and bystander is the most important factor in early arrival. Additionally, we found that knowledge about thrombolysis and transportation by ambulance may shorten prehospital delay. Therefore, widespread and repeated public education via the media is needed to improve recognition of stroke symptoms and ensure the appropriate response. Furthermore, baseline surveys of public awareness of stroke should also be carried out to evaluate current knowledge.

\begin{abstract}
Acknowledgements and funding
This study was supported by grants from the Korea Health 21 R\&D Project, Ministry of Health and Welfare, Republic of Korea (A060171). The funding organization had no role in the design, conduct, analysis, or preparation of this report.

Dr. Byung-Woo Yoon received research grants from the Ministry of Health and Welfare, Republic of Korea to conduct this study.
\end{abstract}

\section{Author details}

${ }^{1}$ Department of Neurology, Hanyang University Hospital, Seoul, Republic of Korea. ${ }^{2}$ Department of Neurology, Seoul National University Hospital, Seoul, Republic of Korea. ${ }^{3}$ Department of Neurology, Seoul National University Bundang hospital, Seongnam, Republic of Korea. ${ }^{4}$ Department of Neurology, St. Mary's hospital, Seoul Korea. ${ }^{5}$ Department of Neurology, Inje University Ilsan Paik Hospital, Goyang, Republic of Korea. ${ }^{6}$ Department of Neurology, Yonsei University Severance Hospital, Seoul, Republic of Korea. ${ }^{7}$ Department of Neurology, Eulji General Hospital, Seoul, Republic of Korea. ${ }^{8}$ Department of Neurology, Dongguk University International Hospital, Koyang, Republic of Korea. ${ }^{9}$ Department of Neurology, Konkuk University Hospital, Seoul, Republic of Korea. ${ }^{10}$ Department of Neurology, Samsung medical center, Seoul, Republic of Korea. ${ }^{11}$ Department of Neurology, Asan medical center, University of Ulsan, Seoul, Republic of Korea. ${ }^{12}$ Department of Neurology, Seoul National University Boramae hospital, Seoul, Republic of Korea. ${ }^{13}$ Department of Neurology, Hallym University Sacred Hospital, Anyang, Republic of Korea. ${ }^{14}$ Department of Neurology, Soonchunhyang University Hospital, Seoul, Republic of Korea. ${ }^{15}$ Department of Neurology, Seoul-Daehyo Hospital, Seoul, Republic of Korea. ${ }^{16}$ Department of Neurology, Inha University Hospital, Incheon, Republic of Korea.

\section{Authors' contributions}

YSK interpreted the data and drafted the manuscript. SSP, KK, SL and SHL participated in the design of the study and intellectual discussion of the results. HJB, AHC, YJC, MKH, JHH, DEK, HYK, GMK, SUK, HMK, BCL, KBL, YSL,
HSN, MSO, JMP, JHR and KHY are principle investigators of each hospital. They participated in the study design and competitively recruited patients. BWY conceived the study, and participated in its design and coordination. He recruited patients, contributed to the intellectual discussion of the concept of the article and gave final approval of the version to be published. All authors read and approved the final manuscript. Each author has participated sufficiently in the work to take public responsibility for appropriate portions of the content.

\section{Competing interests}

The authors declare that they have no competing interests.

Received: 17 September 2010 Accepted: 6 January 2011 Published: 6 January 2011

\section{References}

1. Hacke W, Kaste M, Bluhmki E, Brozman M, Davalos A, Guidetti D, Larrue V, Lees KR, Medeghri Z, Machnig T, et al: Thrombolysis with alteplase 3 to 4.5 hours after acute ischemic stroke. $N$ Engl J Med 2008, 359:1317-1329.

2. Reeves MJ, Arora S, Broderick JP, Frankel M, Heinrich JP, Hickenbottom S, Karp H, LaBresh KA, Malarcher A, Mensah G, et al: Acute stroke care in the US: results from 4 pilot prototypes of the Paul Coverdell National Acute Stroke Registry. Stroke 2005, 36:1232-1240.

3. Yu KH BH, Kwon SU, Kang DW, Hong KS, Lee YS, Rha JH, Koo JS, Kim JS, Kim JH, Lee JH, Cho SJ, Hwang SH, Jung S, Han MK, Cho KH, Kim BC, Shin DJ, Chang DI, Park JH, Kim EG, Jung DS, Ahn MY, Lee DH, Park KW, Kim YJ, Lee KY, Heo JH, Kim SH, Lee KH, Chung CS, Cha JK, Lee JH, Uhm KY, Lee BC, Roh JK: Analysis of 10,811 Cases with Acute Ischemic Stroke from Korean Stroke Registry: Hospital-Based Multicenter Prospective Registration Study. J Korean Neurol Assoc 2006, 24:535-543.

4. Harraf F, Sharma AK, Brown MM, Lees KR, Vass RI, Kalra L: A multicentre observational study of presentation and early assessment of acute stroke. BMJ 2002, 325:17.

5. Kwan J, Hand P, Sandercock P: A systematic review of barriers to delivery of thrombolysis for acute stroke. Age Ageing 2004, 33:116-121.

6. Mandelzweig L, Goldbourt U, Boyko V, Tanne D: Perceptual, social, and behavioral factors associated with delays in seeking medical care in patients with symptoms of acute stroke. Stroke 2006, 37:1248-1253.

7. Kim JS, Yoon SS: Perspectives of stroke in persons living in Seoul, South Korea. A survey of 1000 subjects. Stroke 1997, 28:1165-1169.

8. Derex L, Adeleine P, Nighoghossian N, Honnorat J, Trouillas P: Factors influencing early admission in a French stroke unit. Stroke 2002, 33:153-159.

9. Wester P, Radberg J, Lundgren B, Peltonen M: Factors associated with delayed admission to hospital and in-hospital delays in acute stroke and TIA: a prospective, multicenter study. Seek- Medical-Attention-in-Time Study Group. Stroke 1999, 30:40-48. 
10. Williams LS, Bruno A, Rouch D, Marriott DJ: Stroke patients' knowledge of stroke. Influence on time to presentation. Stroke 1997, 28:912-915.

11. Barr J, McKinley S, O'Brien E, Herkes G: Patient recognition of and response to symptoms of TIA or stroke. Neuroepidemiology 2006, 26:168-175.

12. Shah M, Makinde KA, Thomas P: Cognitive and behavioral aspects affecting early referral of acute stroke patients to hospital. J Stroke Cerebrovasc Dis 2007, 16:71-76.

13. Chang KC, Tseng MC, Tan TY: Prehospital delay after acute stroke in Kaohsiung, Taiwan. Stroke 2004, 35:700-704.

14. Morris DL, Rosamond W, Madden K, Schultz C, Hamilton S: Prehospital and emergency department delays after acute stroke: the Genentech Stroke Presentation Survey. Stroke 2000, 31:2585-2590.

15. Memis S, Tugrul E, Evci ED, Ergin F: Multiple causes for delay in arrival at hospital in acute stroke patients in Aydin, Turkey. BMC Neurol 2008, 8:15.

16. Inatomi Y, Yonehara T, Hashimoto Y, Hirano T, Uchino M: Pre-hospital delay in the use of intravenous rt-PA for acute ischemic stroke in Japan. J Neurol Sci 2008, 270:127-132.

17. Lacy CR, Suh DC, Bueno M, Kostis JB: Delay in presentation and evaluation for acute stroke: Stroke Time Registry for Outcomes Knowledge and Epidemiology (S.T.R.O.K.E.). Stroke 2001, 32:63-69.

18. Maestroni A, Mandelli C, Manganaro D, Zecca B, Rossi P, Monzani V, Torgano G: Factors influencing delay in presentation for acute stroke in an emergency department in Milan, Italy. Emerg Med J 2008, 25:340-345.

19. Jorgensen HS, Nakayama H, Reith J, Raaschou HO, Olsen TS: Factors delaying hospital admission in acute stroke: the Copenhagen Stroke Study. Neurology 1996, 47:383-387.

20. Smith MA, Doliszny KM, Shahar E, McGovern PG, Arnett DK, Luepker RV: Delayed hospital arrival for acute stroke: the Minnesota Stroke Survey. Ann Intern Med 1998, 129:190-196.

21. Rosamond WD, Gorton RA, Hinn AR, Hohenhaus SM, Morris DL: Rapid response to stroke symptoms: the Delay in Accessing Stroke Healthcare (DASH) study. Acad Emerg Med 1998, 5:45-51.

22. Howard VJ, Lackland DT, Lichtman JH, McClure LA, Howard G, Wagner L, Pulley L, Gomez CR: Care seeking after stroke symptoms. Ann Neurol 2008, 63:466-472.

\section{Pre-publication history}

The pre-publication history for this paper can be accessed here: http://www.biomedcentral.com/1471-2377/11/2/prepub

doi:10.1186/1471-2377-11-2

Cite this article as: Kim et al:: Stroke awareness decreases prehospital delay after acute ischemic stroke in korea. BMC Neurology 2011 11:2.

\section{Submit your next manuscript to BioMed Central and take full advantage of:}

- Convenient online submission

- Thorough peer review

- No space constraints or color figure charges

- Immediate publication on acceptance

- Inclusion in PubMed, CAS, Scopus and Google Scholar

- Research which is freely available for redistribution

Submit your manuscript at www.biomedcentral.com/submit
C Biomed Central 